\title{
Extracorporeal shock wave therapy versus local corticosteroid injection for the treatment of carpal tunnel syndrome: a meta-analysis
}

Wenhao $\mathrm{Li}^{1,2 \dagger}$, Chunke Dong ${ }^{1,2+}$, Hongyu Wei ${ }^{2}$, Zhencheng Xiong ${ }^{3}$, Liubo Zhang ${ }^{1,2}$, Jun Zhou ${ }^{1,2}$, Yanlei Wang ${ }^{1,2}$, Jipeng Song ${ }^{2,4}$ and Mingsheng $\operatorname{Tan}^{2^{*}}$ (D)

\begin{abstract}
Background: Many studies have demonstrated the effectiveness of extracorporeal shock wave therapy (ESWT) and local corticosteroid injection (LCI) for the treatment of carpal tunnel syndrome (CTS), and some studies showed that the effect of ESWT was superior to $\mathrm{LCl}$. We performed this meta-analysis to compare the clinical effects across the two therapies.

Methods: Relevant randomized controlled trials (RCTs) comparing ESWT and LCI for the treatment of CTS were searched in electronic database. The Cochrane risk bias tool was used for quality assessment. After data extraction and quality assessment of the included studies, a meta-analysis was performed using RevMan 5.3 software. Mean differences (MDs), odds ratios (ORs), and 95\% confidence intervals (Cls) were analyzed. The protocol for this systematic review was registered on INPLASY (202080025) and is available in full on the inplasy.com (https://doi. org/10.37766/inplasy2020.8.0025)

Results: A total of 5 RCT studies with 204 patients were included from the electronic database. The meta-analysis results showed that two therapies were not significantly different in terms of visual analog scale (VAS) score $(P=$ 0.65), Boston Carpal Tunnel Questionnaire (BQ) score $(P=0.14)$, sensory distal latency $(P=0.66)$, and nerve conduction velocity ( $\mathrm{NCV}$ ) of the sensory nerve $(P=0.06)$. There were significant differences between the results of motor distal latency $(P<0.0001)$, compound muscle action potential (CMAP) amplitude $(P<0.00001)$, and sensory nerve action potential (SNAP) amplitude $(P=0.004)$.

Conclusions: In terms of pain relief and function improvement, the effects of ESWT and LCl are not significantly different. In terms of electrophysiological parameters, LCI has a stronger effect on shortening motor distal latency; ESWT is superior to $\mathrm{LCl}$ in improving action potential amplitude. ESWT is a noninvasive treatment with fewer complications and greater patient safety. In light of the heterogeneity and limitations, these conclusions require further research for definitive conclusions to be drawn.
\end{abstract}

Keywords: Carpal tunnel syndrome, Extracorporeal shock wave, Local corticosteroid injection, Meta-analysis

\footnotetext{
*Correspondence: zrtanms@163.com

'Wenhao Li and Chunke Dong contributed equally to this study and should be considered co-first authors.

${ }^{2}$ Department of Orthopaedic Surgery, China-Japan Friendship Hospital, Beijing 100029, China

Full list of author information is available at the end of the article
}

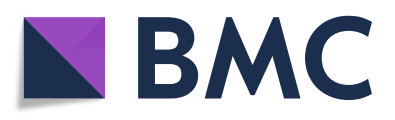

(- The Author(s). 2020 Open Access This article is licensed under a Creative Commons Attribution 4.0 International License, which permits use, sharing, adaptation, distribution and reproduction in any medium or format, as long as you give appropriate credit to the original author(s) and the source, provide a link to the Creative Commons licence, and indicate if changes were made. The images or other third party material in this article are included in the article's Creative Commons licence, unless indicated otherwise in a credit line to the material. If material is not included in the article's Creative Commons licence and your intended use is not permitted by statutory regulation or exceeds the permitted use, you will need to obtain permission directly from the copyright holder. To view a copy of this licence, visit http://creativecommons.org/licenses/by/4.0/. The Creative Commons Public Domain Dedication waiver (http://creativecommons.org/publicdomain/zero/1.0/) applies to the data made available in this article, unless otherwise stated in a credit line to the data. 


\section{Introduction}

Carpal tunnel syndrome (CTS) is the most common and widely studied nerve entrapment syndrome [1], accounting for approximately $90 \%$ of all compressive neuropathies [2]. It is caused by compression of the median nerve while it passes through the carpal tunnel, a limited space. Inflammation, edema, tendon spasm, hormone level changes, and physical activity are all associated with increased nerve compression, causing pain and numbness. In severe cases, muscle weakness in muscles innervated by the median nerve may occur [3]. Based on differing diagnostic criteria, the reported prevalence and incidence of CTS can vary greatly. The use of clinical criteria in diagnosis is more common than the use of electrophysiological criteria. It is generally estimated that $10 \%$ of people suffer from CTS at some point in their lives [3]. Additional studies have shown that the incidence and prevalence in middle-aged populations are $0.125-1 \%$ and $5-16 \%$, respectively. Various risk factors reportedly include high body mass index, female gender, age, and pregnancy, among others [2]. CTS is also recognized as one of the most prevalent occupational health injuries, particularly in industries where work involves high-intensity, repetitive use of the wrists, and vibration tools. The incidence rate in workers employed in such industries is approximately 5\% [2]. Treatment is usually organized into conservative treatment and surgical treatment. The overall therapeutic goals are generally to relieve symptoms, improve function, and prevent further nerve damage. More common treatment methodologies include local corticosteroid injection (LCI) and carpal tunnel release surgery [4].

Extracorporeal shock wave therapy (ESWT) was initially used clinically as a lithotripsy method to destroy calcified deposits in the body, especially stones in the kidney, bile duct, and salivary gland ducts. Over the past 30 years, this technology has been increasingly applied to various musculoskeletal diseases, such as shoulder calcified tendinitis, delayed fracture healing, and others [5]. Many studies, including some randomized controlled trials (RCTs) that have demonstrated the effectiveness of ESWT for the treatment of CTS, and the effect of ESWT is superior to LCI [6-15]. We performed this metaanalysis of related RCTs to compare the efficacy of ESWT and LCI to provide greater evidence for clinical decision-making.

\section{Methods}

This meta-analysis was performed in accordance with the Preferred Reporting Items for Systematic Reviews and Meta-Analyses (PRISMA) statement [16] and the Cochrane Handbook [17]. Ethical approval was not required since this is a meta-analysis of published studies.

\section{Inclusion and exclusion criteria}

All studies included in this meta-analysis met the following criteria: (1) published clinical RCT; (2) patients with a clear diagnosis of CTS, and the age, gender, and nationality were not limited; and (3) ESWT was used as an intervention measure, and CTS was used as a control measure, and complete comparison data between ESWT and CTS could be obtained.

Studies were excluded according to the following exclusion criteria: (1) CTS caused by trauma, fracture, tumor, infection, endocrine system disease, etc., or combined with diabetes, peripheral polyneuropathy, coagulation disorder, thrombosis, mental system disease, etc.; (2) patients who had received carpal tunnel surgery; (3) patients who had received oral hormones, non-steroidal anti-inflammatory drugs, and splint fixation before being enrolled; and (4) animal experiments.

\section{Search strategies}

A systematic computer-based retrieval was performed on the literatures published before September 1, 2020, in PubMed, Embase, Cochrane Library, China National Knowledge Infrastructure database (CNKI), WanFang database, and Chinese Scientific Journal Database. The following search terms were used: "extracorporeal shock wave", "local corticosteroid injection", "injection", "carpal tunnel syndrome" with the Boolean operators "AND" or "OR". At the same time, we traced the references of the included literatures and the meta-analysis related to this research, screened, and evaluated the references to determine potential researches.

\section{Data extraction}

Two researchers conducted the literature search, and strictly followed the inclusion and exclusion criteria for the preliminary screening and secondary screening of the literatures. After the screening, two independent researchers extracted data from the literatures that met the requirements and then the data were checked by the third researcher. Regarding any differences in the included literatures, consensus was reached through discussion among all researchers. The missing data in the literatures had been completed by contacting the authors by email. The main data extracted in this study include name of the first author, year of publication, sample size of the experimental group and the control group, gender ratio of patients, average age, intervention methods and treatment frequency, country, study design type, followup time, and outcome indicators. The extracted data had been reviewed to ensure accuracy.

\section{Quality assessment}

This study used the Cochrane risk bias tool [18] for quality evaluation. This tool includes evaluations in 
seven aspects: random sequence generation, allocation hiding, blinding of participants and implementers, blinding of outcome evaluators, incomplete outcome data, selective reporting, and other biases. The risk of bias in each area is judged as low risk, high risk, or unknown risk. The quality of the studies was evaluated by two researchers.

\section{Data analysis}

The Review Manager software (RevMan 5.3) was used for statistical analysis. Continuous variables were reported as mean difference (MD) and 95\% confidence interval $(\mathrm{CI})$, while dichotomy variables were reported as odds ratio (OR) and 95\% CI. Statistical heterogeneity was judged by the combination of $Q$ value statistics and $I^{2}$ statistics. The larger the $I^{2}$, the greater the heterogeneity. If there was heterogeneity in the study $\left(I^{2} \geq 50 \%\right)$, the random effects model was adopted; otherwise, the fixed effects model was adopted $\left(I^{2}<\right.$ $50 \%)$. The extracted data was input into the computer, reviewed, and independently analyzed by two researchers.

\section{Results}

\section{Search result}

A total of 93 related studies were confirmed from the electronic database. After deleting duplicate studies, 83 studies were obtained. After careful full-text evaluation of these studies according to the inclusion and exclusion criteria, 5 RCT studies [11-15] with 204 patients were included in the final comprehensive analysis. The literature screening flow diagram is shown in Fig. 1, and the basic characteristics of the included studies are shown in Table 1.

\section{Quality assessment}

Among five analyzed RCTs, Xu et al.'s study used a computer to generate a list of random numbers and then generated a random sequence; patients were grouped using sealed envelopes, the test result evaluator was unaware of the grouping situation, there was no withdrawal or loss to follow-up, and the dataset was complete. The study of Atthakomol et al. used a random number generator to generate random sequences and further used envelopes for grouping; however, whether the envelopes were sealed was not stated. The evaluator of the test

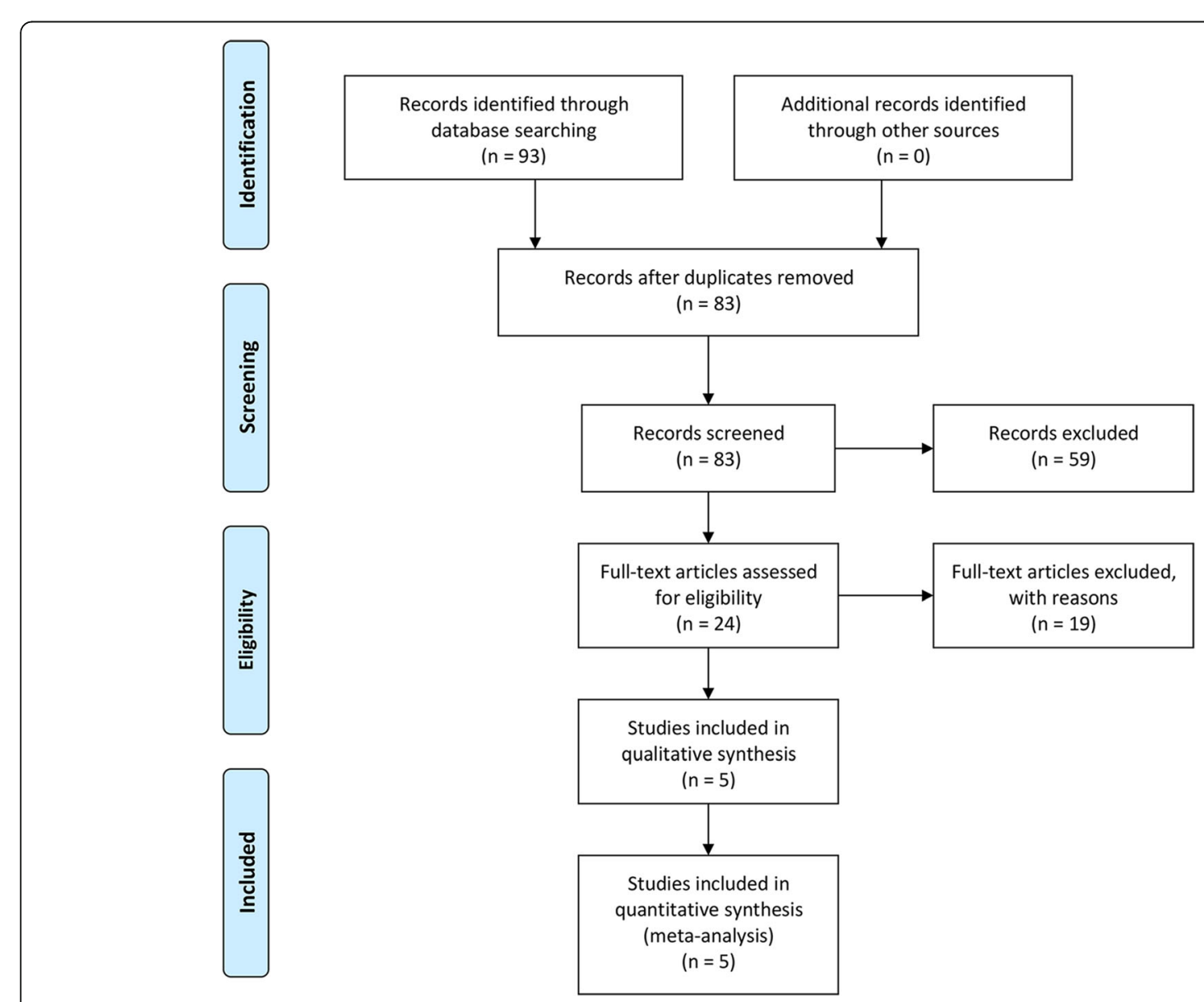

Fig. 1 Flow diagram of the study selection process 


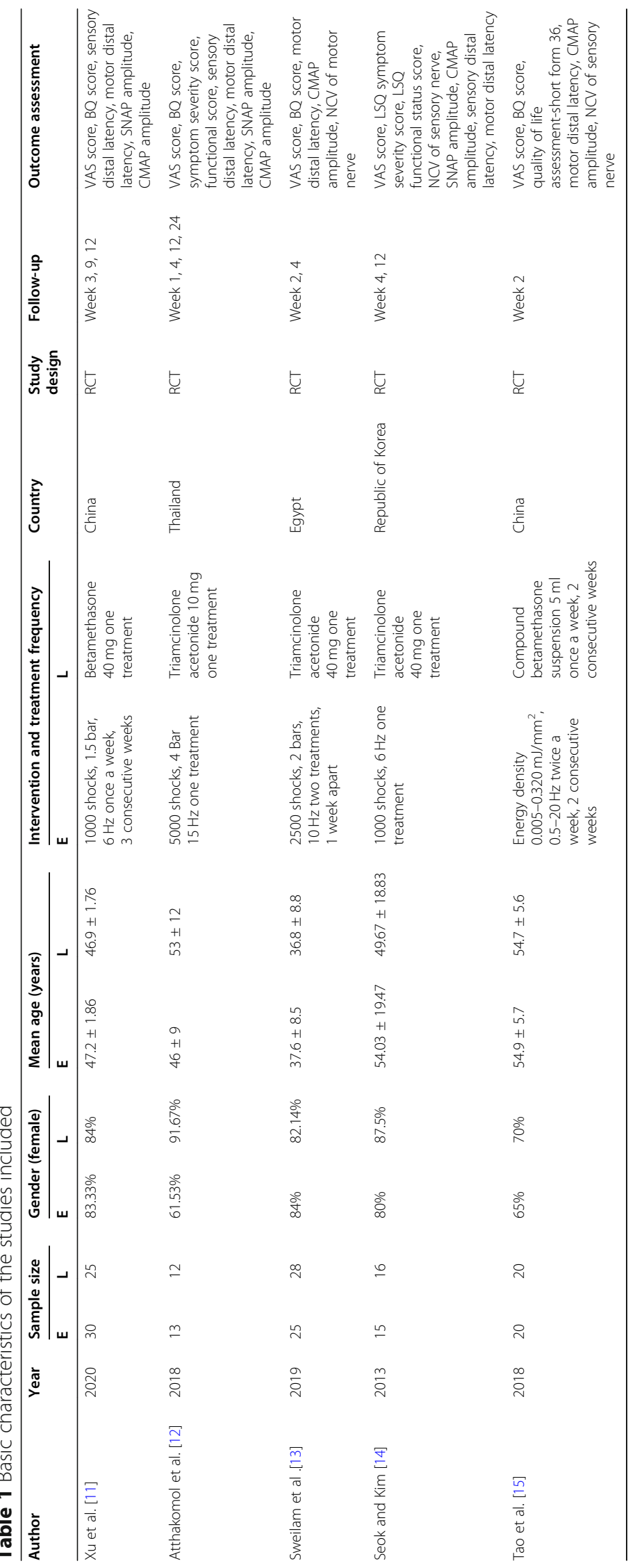




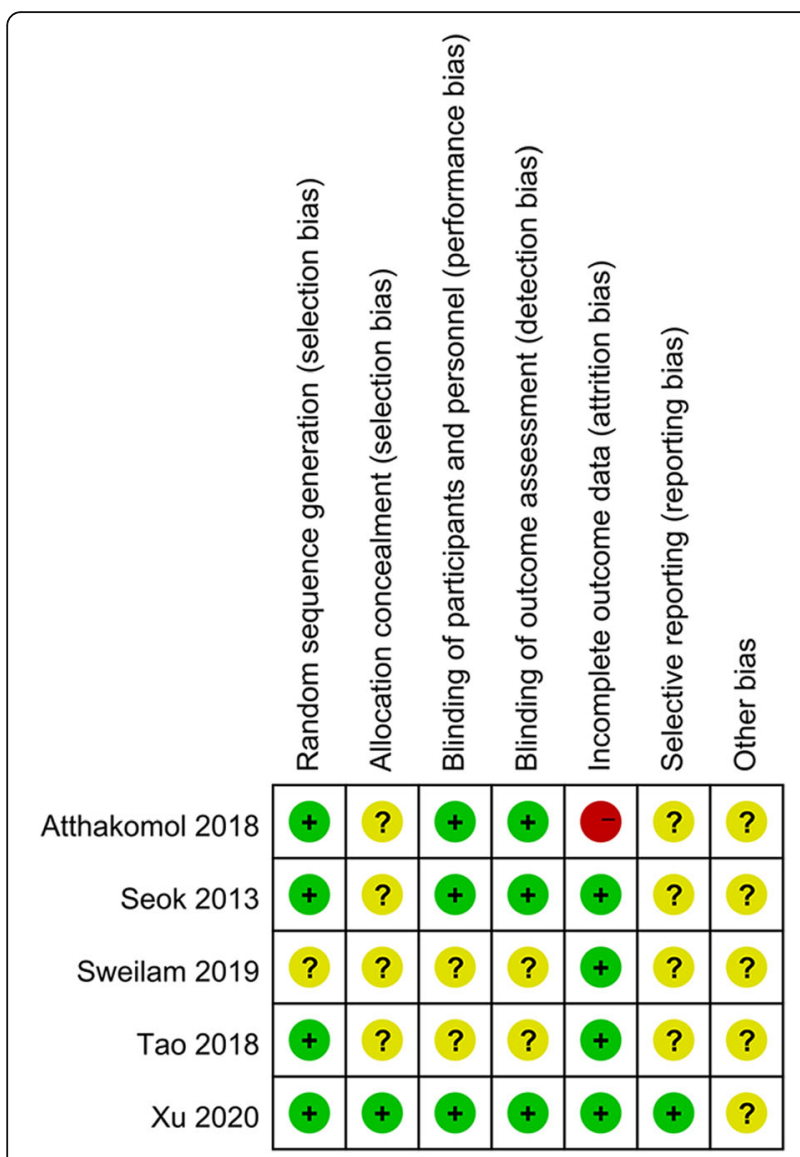

Fig. 2 Risk of bias summary. Plus sign indicates low risk of bias. minus sign high risk ofbias, and question mark bias unclear

results was unaware of the results, and several patients withdrew or were lost to follow-up. In the study by Sweilam et al., the random sequence generation, allocation hiding, and blinding methodology were not provided. The study of Seok and Kim used random number generation software to generate random sequences. The allocation method was not specified; the test result evaluator was unaware of the results, and there was no withdrawal or loss to follow-up. Tao et al.'s study used a random number table method to generate random sequences. There was no withdrawal or loss to followup, but the remaining factors were not explained. As shown in Fig. 2.

\section{Comparison of the visual analog scale (VAS) scores for ESWT and LCI}

The VAS scores of ESWT and LCI were compared in five studies [11-15], including 202 patients (101 patients received ESWT treatment and 101 patients received LCI treatment), as shown in Fig. 3. The heterogeneity test showed that there was heterogeneity between the studies $\left(P<0.0001, I^{2}=84 \%\right)$, so the random effects model was used to analyze the data of the five studies. The comprehensive results showed that the difference between the ESWT group and the LCI group was not statistically significant (MD $-0.22,95 \% \mathrm{CI}-1.16$ to $0.72, P=0.65$ ).

\section{Comparison of the Boston Carpal Tunnel Questionnaire (BQ) scores for ESWT and LCI}

The BQ scores of ESWT and LCI were compared in three included studies [11-13], including 131 patients (66 patients received ESWT treatment and 65 patients received LCI treatment), as shown in Fig. 4. The heterogeneity test showed that there was heterogeneity between the studies $\left(P<0.00001, I^{2}=97 \%\right)$, so the random effects model was used to analyze the data of three studies. The comprehensive results showed that the difference between the ESWT group and the LCI group was not statistically significant (MD $-5.69,95 \% \mathrm{CI}-13.26$ to 1.88, $P=0.14$ ).

\section{Comparison of the sensory distal latency for ESWT and LCI}

The sensory distal latency of ESWT and LCI was compared in three included studies [11, 12, 14], including 103 patients (55 patients received ESWT treatment, 53 patients received LCI treatment), as shown in Fig. 5. The heterogeneity test showed that there was heterogeneity between the studies ( $P=0.0002, I^{2}=88 \%$ ), so the random effects model was used to analyze the data of the three studies. The comprehensive results showed that

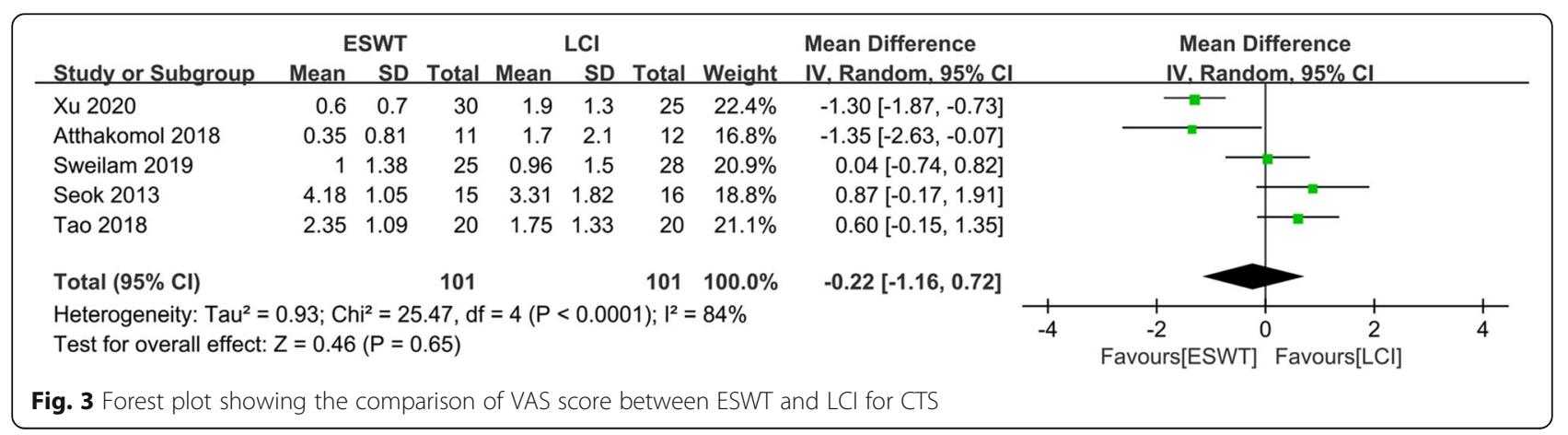




\begin{tabular}{|c|c|c|c|c|c|c|c|c|c|c|c|}
\hline Study or Subgroup & $\begin{array}{r}\text { ESWT } \\
\text { Mean SD } \\
\end{array}$ & Total & Mean & $\begin{array}{r}\mathrm{LCl} \\
\mathrm{SD} \\
\end{array}$ & Total & Weight & $\begin{array}{l}\text { Mean Difference } \\
\text { IV. Random, } 95 \% \mathrm{Cl}\end{array}$ & & $\begin{array}{l}\text { Mean Dif } \\
\text { IV. Randor }\end{array}$ & $\begin{array}{l}\text { fference } \\
\mathrm{m}, 95 \% \mathrm{Cl}\end{array}$ & \\
\hline Atthakomol 2018 & $22.3 \quad 2.7$ & 30 & 31.8 & 3.4 & 25 & $37.0 \%$ & $-9.50[-11.15,-7.85]$ & & - & & \\
\hline Sweilam 2019 & $24 \quad 4.7$ & 11 & 32 & 14 & 12 & $25.8 \%$ & $-8.00[-16.39,0.39]$ & & 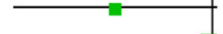 & & \\
\hline Xu 2020 & $12.3 \quad 2.1$ & 25 & 12.6 & 3.1 & 28 & $37.2 \%$ & $-0.30[-1.71,1.11]$ & & & & \\
\hline Total $(95 \% \mathrm{Cl})$ & & 66 & & & 65 & $100.0 \%$ & $-5.69[-13.26,1.88]$ & & & & \\
\hline $\begin{array}{l}\text { Heterogeneity: } \mathrm{Tau}^{2} \\
\text { Test for overall effect: }\end{array}$ & $\begin{array}{l}39.60 ; \mathrm{Chi}^{2}= \\
\mathrm{Z}=1.47(\mathrm{P}=\end{array}$ & $\begin{array}{l}=69.87, \\
=0.14)\end{array}$ & $d f=2($ & $(P<0$ & .00001 & 1); $\left.\right|^{2}=97 \%$ & & -20 & $\begin{array}{cc} & -10 \\
\text { Favours [ESWT] }\end{array}$ & Favours $[\mathrm{LCl}]$ & 20 \\
\hline
\end{tabular}

the difference between the ESWT group and the LCI group was not statistically significant (MD 0.18, 95\%CI -0.62 to $0.97, P=0.66)$.

Comparison of the motor distal latency for ESWT and LCI The motor distal latency of ESWT and LCI was compared in five included studies [11-15], including 201 patients (100 patients received ESWT treatment, 101 patients received LCI treatment), as shown in Fig. 6. The heterogeneity test showed that there was no heterogeneity between the studies $\left(P=0.14, I^{2}=43 \%\right)$, so the fixed effects model was used to analyze the data of the five studies. The comprehensive results showed that the difference between the ESWT group and the LCI group was statistically significant (MD $0.17,95 \%$ CI 0.10 to $0.25, P<0.0001)$.

\section{Comparison of the compound muscle action potential (CMAP) amplitude for ESWT and LCI}

The CMAP amplitudes of ESWT and LCI were compared in five included studies [11-15], including 201 patients (100 patients received ESWT treatment and 101 patients received LCI treatment), as shown in Fig. 7. The heterogeneity test showed that there was no heterogeneity between the studies $\left(P=0.15, I^{2}=41 \%\right)$, so the fixed effects model was used to analyze the data of the five studies. The comprehensive results showed that the difference between the ESWT group and the LCI group was statistically significant (MD $-0.48,95 \% \mathrm{CI}-0.61$ to $-0.35, P<0.00001)$.
Comparison of the sensory nerve action potential (SNAP) amplitude for ESWT and LCI

The SNAP amplitudes of ESWT and LCI were compared in three included studies [11, 12, 14], including 108 patients (55 patients received ESWT treatment, 53 patients received LCI treatment), as shown in Fig. 8. The heterogeneity test showed that there was no heterogeneity between the studies $\left(P=0.81, I^{2}=0 \%\right)$, so the fixed effects model was used to analyze the data of the three studies. The comprehensive results showed that the difference between the ESWT group and the LCI group was statistically significant (MD $-1.56,95 \% \mathrm{CI}-2.62$ to $-0.50, P=0.004)$.

\section{Comparison of the nerve conduction velocity (NCV) of sensory nerve for ESWT and LCI}

The NCV of sensory nerve of ESWT and LCI were compared in two included studies $[14,15]$, including $71 \mathrm{pa-}$ tients (35 patients received ESWT treatment, 36 patients received LCI treatment), as shown in Fig. 9. The heterogeneity test showed that there was no heterogeneity between the studies $\left(P=0.53, I^{2}=0 \%\right)$, so the fixed effects model was used to analyze the data of the two studies. The comprehensive results showed that the difference between the ESWT group and the LCI group was not statistically significant $(\mathrm{MD}-2.33,95 \% \mathrm{CI}-4.77$ to 0.11 , $P=0.06)$.

\section{Publication bias}

VAS score was the common outcome index of five RCT studies, and it was also the main indicator for CTS symptoms. Therefore, this outcome index was used to

\begin{tabular}{|c|c|c|c|c|c|c|c|c|c|}
\hline \multirow[b]{2}{*}{ Study or Subgroup } & \multicolumn{2}{|c|}{ ESWT } & \multicolumn{3}{|r|}{ LCI } & \multirow[b]{2}{*}{ Total } & \multirow[b]{2}{*}{ Weight } & \multirow{2}{*}{$\begin{array}{l}\text { Mean Difference } \\
\text { IV, Random, } 95 \% \mathrm{CI}\end{array}$} & \multirow{2}{*}{$\begin{array}{c}\text { Mean Difference } \\
\text { IV, Random, } 95 \% \mathrm{CI}\end{array}$} \\
\hline & Mean & SD & Total & Mean & SD & & & & \\
\hline Xu 2020 & 4 & 0.08 & 30 & 4.4 & 0.07 & 25 & $39.6 \%$ & $-0.40[-0.44,-0.36]$ & $\mathbf{\square}$ \\
\hline Atthakomol 2018 & 4.1 & 0.74 & 10 & 3.6 & 1.3 & 12 & $27.0 \%$ & $0.50[-0.37,1.37]$ & \\
\hline Seok 2013 & 3.88 & 0.64 & 15 & 3.28 & 0.9 & 16 & $33.4 \%$ & $0.60[0.05,1.15]$ & - \\
\hline Total $(95 \% \mathrm{Cl})$ & & & 55 & & & 53 & $100.0 \%$ & $0.18[-0.62,0.97]$ & \\
\hline \multicolumn{9}{|c|}{$\begin{array}{l}\text { Heterogeneity: } \mathrm{Tau}^{2}=0.42 ; \mathrm{Chi}^{2}=16.85, \mathrm{df}= \\
\text { Test for overall effect: } \mathrm{Z}=0.44(\mathrm{P}=0.66)\end{array}$} & $\begin{array}{ccccc}-2 & -1 & 0 & 1 & 2 \\
\text { Favours [ESWT] } & \text { Favours [LCl] }\end{array}$ \\
\hline
\end{tabular}




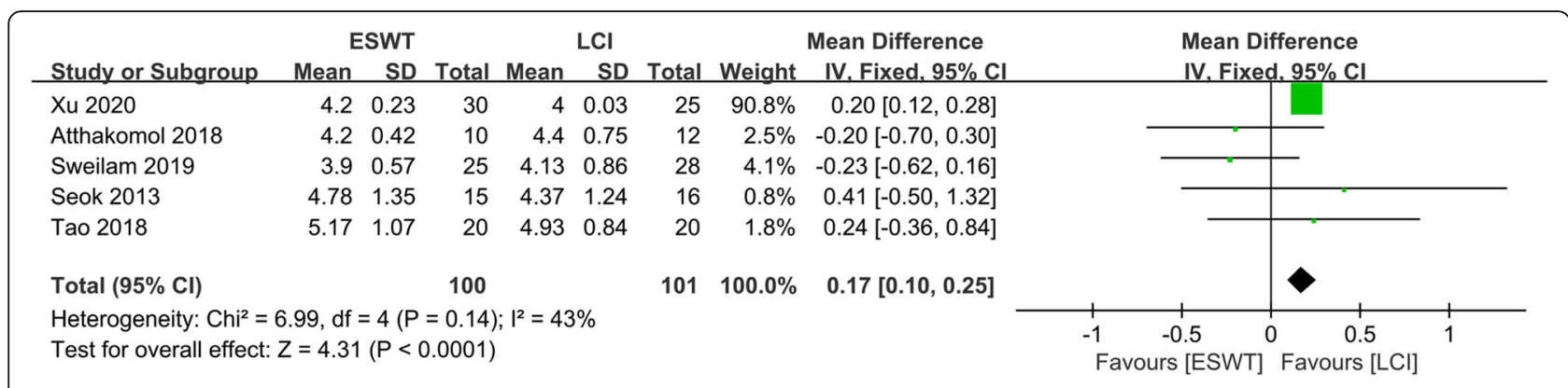

Fig. 6 Forest plot showing the comparison of motor distal latency between ESWT and LCI for CTS

make a funnel plots to detect publication bias, as shown in Fig. 10. Visual inspection of the funnel plots showed symmetry, suggesting that there was no publication bias.

\section{Sensitivity analysis}

For VAS score $\left(I^{2}=84 \%\right)$, BQ score $\left(I^{2}=97 \%\right)$ and sensory distal latency $\left(I^{2}=88 \%\right)$, the sensitivity was tested by eliminating the literature one by one, and found after excluding the study of Xu et al., the $I^{2}$ and $P$ values of the VAS score did not change much, while BQ score and sensory distal latency had no significant heterogeneity $\left(I^{2}=0 \%\right)$, and the differences between groups changed from non-statistically significant before elimination to statistically significant.

\section{GRADE evidence quality evaluation}

The motor distal latency and CMAP amplitude of the two therapies were high-level evidence quality. VAS score, SNAP amplitude, and NCV of sensory nerve were moderate-level evidence quality. BQ score and sensory distal latency were very low-level evidence quality. It indicates that this result still needs to be further confirmed by higher quality RCTs, as shown in Table 2 .

\section{Discussion}

In this study, we identified five RCT studies [11-15] involving 204 total patients to compare the clinical efficacy of ESWT and LCI on CTS. We evaluated seven outcome indicators, of which VAS score and BQ score were the main outcome indicators, while motor distal latency, sensory distal latency, CMAP amplitude, SNAP amplitude, and NCV of the sensory nerve were secondary outcome indicators. Our meta-analysis results showed that the results of the two therapies were not significantly different in terms of VAS score, BQ score, sensory distal latency, and $\mathrm{NCV}$ of the sensory nerve. There were significant differences between the results of the two therapies with respect to motor distal latency, CMAP amplitude, and SNAP amplitude.

Treatment of CTS usually involves splint fixation, LCI, and surgical release. The effectiveness of these methods has been clinically established [19]. The clinical basis of wrist splint fixation is that CTS symptoms appear to improve with rest and are aggravated with activity. The therapeutic effect comes from minimizing carpal tunnel pressure, but there is insufficient evidence for overall effectiveness [20]. Surgery release can quickly relieve any compression, but postoperative complications and delayed return to work may occur [21]. Moreover, some untreated patients will improve spontaneously without surgical intervention. If all CTS patients necessarily undergo carpal tunnel lysis, unnecessary surgeries may be performed [22]. LCI can effectively reduce inflammation and edema of the synovium and tendons, thereby reducing pressure on the median nerve, and can even stabilize the nerve membrane [23]. Its short-term efficacy is strongly supported, and it is therefore the preferred treatment for patients with mild to moderate CTS [24]. Shock waves are defined as a series of acoustic pulses with high pressure peaks, rapid pressure rises,

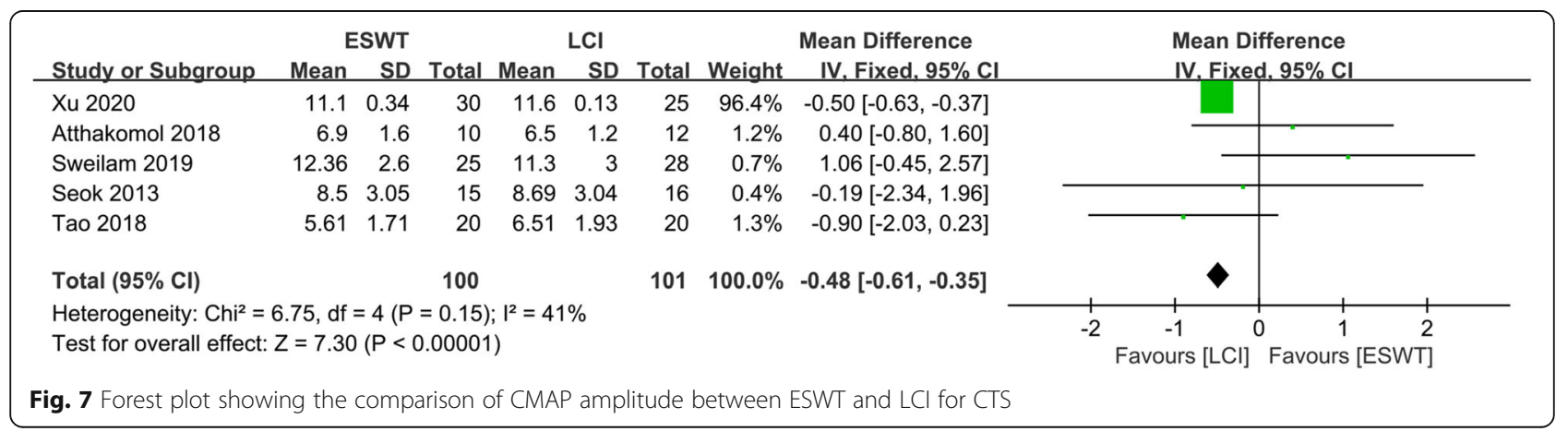




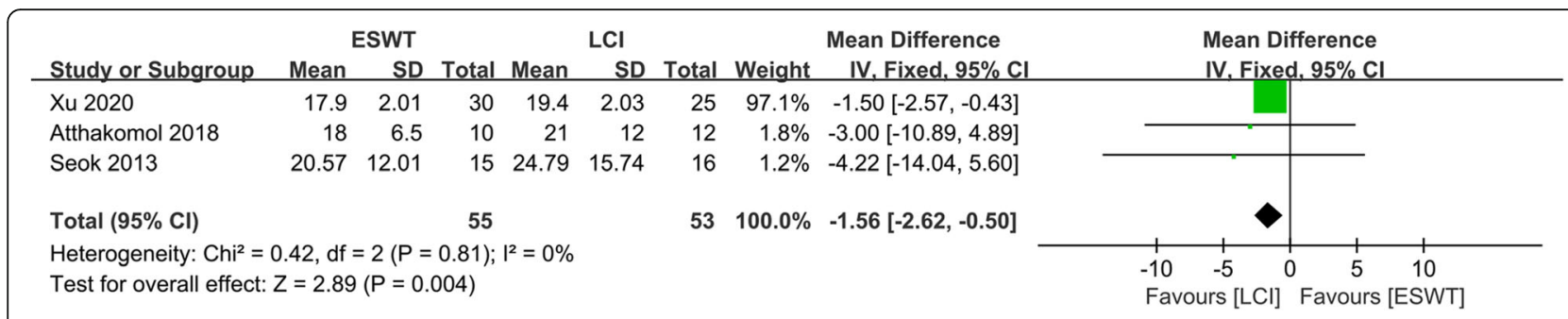

Fig. 8 Forest plot showing the comparison of SNAP amplitude between ESWT and LCI for CTS

and short durations; they are transient pressure disturbances that propagate in three-dimensional space [14]. ESWT is a noninvasive treatment method. It is produced in vitro and is focused on a specific part of the body. It usually uses fluid (water) and coupling gel as a conductive medium for transmission to biological tissues. Its clinical effects have also validated across multiple research studies [6-15].

In the investigated RCT [11-15], ESWT and LCI both showed good clinical efficacy in terms of pain relief, functional improvement, and electrophysiological parameter improvement, which is consistent with the conclusions of the above studies. The purpose of our investigation was to compare the clinical effects across the two therapies, which has been lacking to date. This study is the first meta-analysis to compare the effects of ESWT and LCI for the treatment of CTS. Our results showed that there was no significant difference in the VAS scores and BQ scores between the ESWT and LCI therapies. This shows that, despite the different mechanisms of action, the two therapies are equally effective in relieving pain and improving wrist joint function. LCI relieves pain and improves function by reducing inflammation and edema of the synovium and tendons, reducing nerve compression [23]. Although the mechanism of ESWT in CTS is not yet fully understood, its effects may stem from anti-inflammatory and nerve regeneration mechanisms [9]. Anti-inflammatory effects are seen with both ESWT and LCI, but the mechanism of action of ESWT is different from LCI. The antiinflammatory effect of LCI is achieved by constricting blood vessels in the inflammation site, reducing capillary permeability, and stabilizing the lysosomal membrane, among other effects [23]. In contrast, ESWT stimulates the expression of structural NO synthase in soft tissues in response to pressure, resulting in increased physiologic levels of the powerful inflammation inhibitor NO [25-27]. Additional studies have suggested that the mechanism of pain relief may be caused by a decreased expression of calcitonin gene-related peptide in dorsal root ganglion neurons [28]. ESWT can also increase muscle sensitivity, which helps promote functional recovery [29]. The advantage of LCI is that as a conventional therapy, it is more widely used clinically and its efficacy is supported by more evidence; however, it is an invasive therapy. Due to potential complications and patient tolerance, the frequency and course of LCI treatment are limited. In contrast, ESWT is a noninvasive treatment. Its energy level, time of each treatment, treatment frequency and treatment course can be adjusted according to the patient's condition. Patient tolerance is better, and cumulative clinical efficacy is easier to achieve [21]. ESWT has fewer complications and is safer, which is a major advantage of ESWT over LCI.

In terms of electrophysiological parameters, there was no significant difference between the two treatment groups with respect to NCV of the sensory nerve, but there were significant differences in motor distal latency, CMAP amplitude, and SNAP amplitude. The effect of LCI in shortening motor distal latency was stronger than that of ESWT, and ESWT was superior to LCI for improving SNAP amplitude and CMAP amplitude. Although both treatment methods improve nerve damage and nerve excitability, the mechanisms of the two therapies are different. LCI improves nerve damage by reducing inflammation and edema of the synovium and

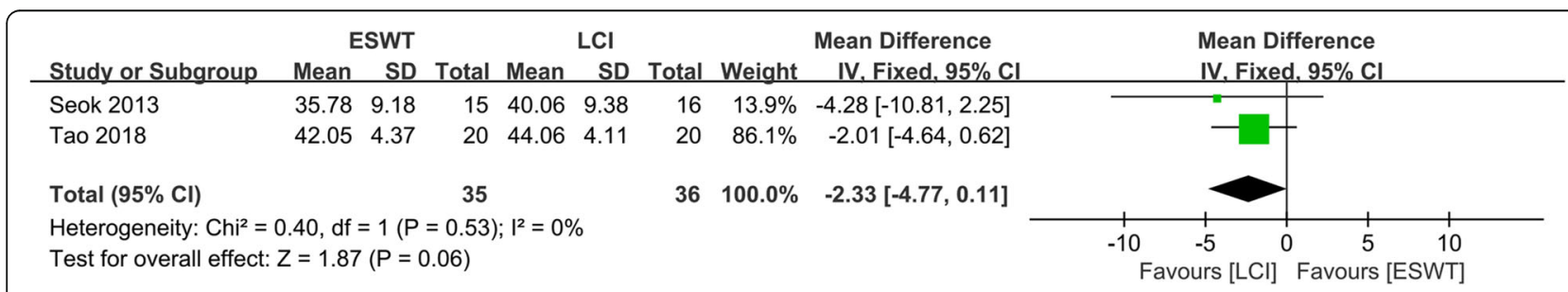

Fig. 9 Forest plot showing the comparison of NCV of sensory nerve between ESWT and LCI for CTS 


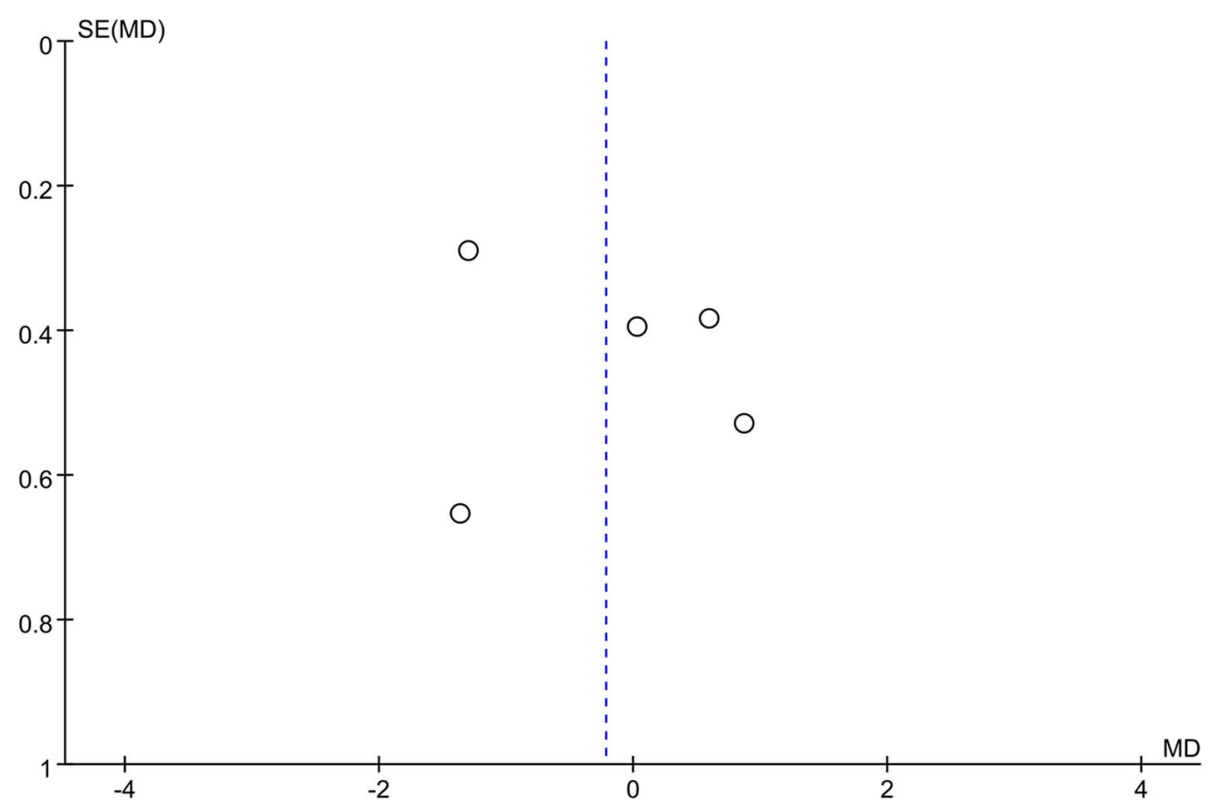

Fig. 10 Funnel plot to detect publication bias for the studies

tendons, thereby reducing nerve compression and stabilizing the nerve membrane [23]. In contrast, ESWT can promote the regeneration of nerve fibers. Animal experiments have shown that ESWT can lead to angiogenesis, tissue repair, neurogenesis, and Schwann cell activation $[29,30]$. ESWT can also increase the rate of axon regeneration. This phenomenon may include faster Wallerian degeneration, enhanced removal of degenerated axons, and a greater ability to regenerate damaged axons [31]. Several studies suggest that this is achieved through direct shock wave effects and indirect cavitation effects, which lead to hematoma formation and focal cell death [5]. It should be noted that most studies suggest that there is no significant relationship between CTS symptom severity score and electrodiagnosis results, and the impact of both intervention measures on electrodiagnosis results appears uncertain [12, 32-34]. The reason may be that electrodiagnosis can only detect large myelinated nerves. The function of small unmyelinated sensory nerves associated with CTS symptoms cannot be assessed by electrodiagnostic measurements. Therefore, neurogenic pain in CTS patients should be processed and treated independently of electrophysiologic data [33]. In the investigated studies, the follow-up time ranged from 1 to 24 weeks. We speculate that it may take longer follow-up observations to fully assess the recovery of the injured nerves and the regaining of complete function. Moreover, the specific parameters of ESWT, such as the number of shocks, energy size, frequency, pressure, and total treatment course, may also be factors that affect subsequent changes in electrophysiological parameters $[5,11]$. This requires further research studies with longer follow-up times to fully evaluate.

None of the five studies we investigated showed significant complications. According to reports from other studies, LCI, as an invasive treatment, has more complications. LCI can adversely affect the function of tendon cells by reducing the synthesis of collagen and proteoglycans and ultimately reduce the mechanical strength of the tendon, leading to further degeneration. Corticosteroids or local anesthetics have also reportedly caused traumatic adventitia damage to peripheral nerve fibers or adventitia cells via hydrostatic pressure, axon and myelin degeneration, intrafascial cavities, tendon rupture, soft tissue atrophy, and accidental nerve damage [35-37]. To date, serious complications of ESWT treatment of CTS have not been reported in the literature $[6-10,12]$. There may reportedly be short-term pain, skin redness, or small hematoma formation after ESWT treatment, but these resolve spontaneously [38].

\section{Limitation}

Our meta-analysis has the following limitations. First, many reported studies did not meet the inclusion criteria. The sample size of the five RCT studies included is relatively small, and the quality of the studies is not sufficiently high, which may influence the overall research conclusions. Second, the included studies are heterogeneous. This may be due to differences in the various ESWT impact times, energy flow density, pressure, frequency, duration of action, and other parameters, as well as differences in LCI drug doses. In addition, the differences in treatment frequency and follow-up times likely 


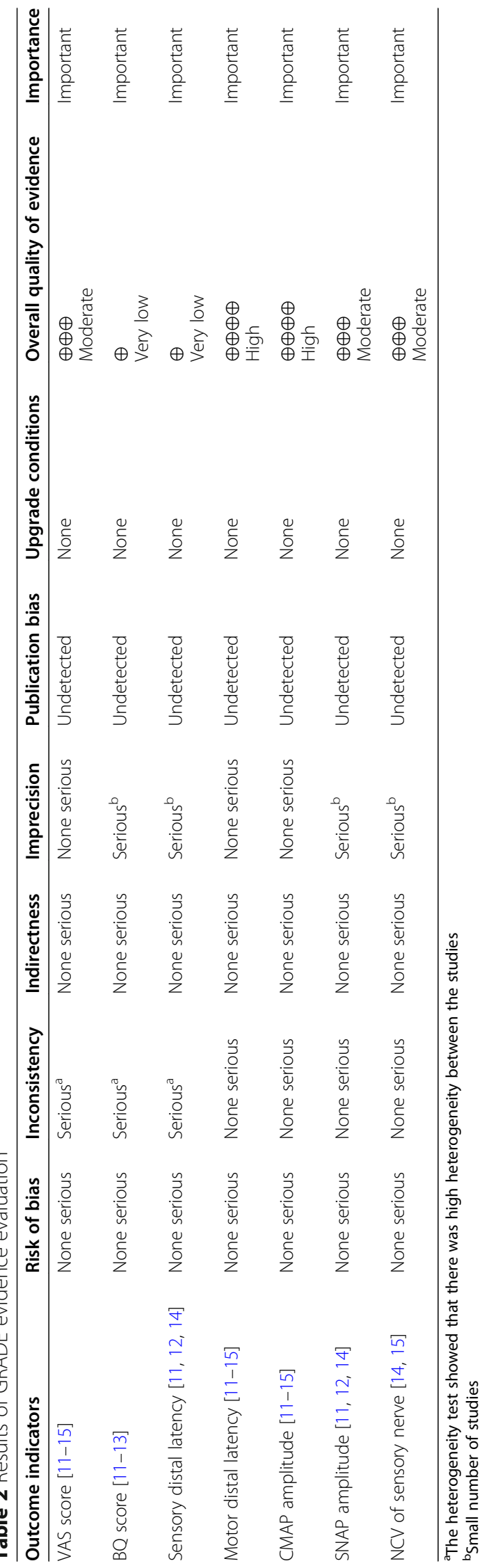


also increase the heterogeneity. Third, the included studies did not have sufficiently lengthy follow-up times. The longest follow-up period was 24 weeks. The long-term efficacy, recurrence rate, and complications associated with the two therapies remains to be definitively investigated and compared [20,39]. Finally, in the process of literature screening, it was found that the outcome indicators selected by each literature were quite different, and the outcome indicators shared by the five studies were few. We hope that the outcome indicators can be unified, so that the comparison results can be more convincing. It can more objectively and truly reflect the treatment effect and changes in the patient's condition. Last but not the least, although our research results show efficacy of both ESWT and LCI, neither of these two therapies can directly relieve compression of the median nerve. For severely affected CTS patients, as well as patients whose symptoms have not improved significantly with treatment or have recurred after treatment, surgery may ultimately be required $[11,20]$.

\section{Conclusion}

In summary, in terms of pain relief and function improvement, the effects of ESWT and LCI for the treatment of CTS are not significantly different. In terms of electrophysiological parameters, the two therapies each have their own advantages. LCI has a stronger effect on shortening motor distal latency; ESWT is superior to LCI in improving action potential amplitude. ESWT is a noninvasive treatment with fewer complications and greater patient safety. However, in light of the heterogeneity and limitations of the present study, these conclusions require further research for definitive conclusions to be drawn.

\section{Supplementary Information}

Supplementary information accompanies this paper at https://doi.org/10. 1186/s13018-020-02082-x.

Additional file 1. Search strategy.

\section{Abbreviations}

ESWT: Extracorporeal shock wave therapy; LCl: Local corticosteroid injection CTS: Carpal tunnel syndrome; RCT: Randomized controlled trials; MD: Mean differences; OR: Odds ratios; Cl: Confidence intervals; VAS: Visual analog scale; BQ: Boston Carpal Tunnel Questionnaire; NCV: Nerve conduction velocity; CMAP: Compound muscle action potential; SNAP: Sensory nerve action potential

\section{Acknowledgements}

Not applicable.

\section{Authors' contributions}

LWH contributed to draft writing and data analysis. DCK designed the study and participated in the data analysis. XZC and ZLB participated in literature screening and data extraction. WYL and SJP contributed to data analysis and charting. WHY and TMS participated in the design of this research and provided guidance and troubleshooting. All authors read and approved the final manuscript. All authors agree to be accountable for all aspects of the work.

Funding

No funds were received in support of this research.

Availability of data and materials

All data generated or analyzed during this study are included in this published article and its supplementary information files.

Ethics approval and consent to participate

This study is a meta-analysis and does not require ethical approval and consent to participate.

\section{Consent for publication}

This research is a meta-analysis of other studies and does not contain any personal information and data, such as name, gender, age, images or videos, etc., so no individual consent is required.

\section{Competing interests}

The authors declare that they have no competing interests in this section.

\section{Author details}

${ }^{1}$ Beijing University of Chinese Medicine, Beijing 100029, China. ${ }^{2}$ Department of Orthopaedic Surgery, China-Japan Friendship Hospital, Beijing 100029, China. ${ }^{3}$ Institute of Medical Technology, Peking University Health Science Center, Peking University Third Hospital, Beijing 100089, China. ${ }^{4}$ Graduate School of Peking Union Medical College, Chinese Academy of Medical Sciences, Beijing 100730, China.

Received: 16 August 2020 Accepted: 10 November 2020

Published online: 23 November 2020

References

1. Olney R. Carpal tunnel syndrome: complex issues with a "simple" condition. Neurology. 2001;56:1431-2. https://doi.org/10.1212/WNL.56.11.1431.

2. Aroori S, Spence RA. Carpal tunnel syndrome. Ulster Med J. 2008;77(1):6-17.

3. Padua L, Coraci D. erra C, et al. Carpal tunnel syndrome: clinical features, diagnosis, and management. Lancet Neurol. 2016;15:1273-84. https://doi. org/10.1016/\$1474-4422(16)30231-9.

4. Gerritsen AAM, de Vet HCW, Scholten RJPM, Bertelsmann FW, de Krom MCTFM, Bouter LM. Splinting vs Surgery in the Treatment of Carpal Tunnel Syndrome: A Randomized Controlled Trial. JAMA. 2002;288(10):1245-51. https://doi.org/10.1001/jama.288.10.1245.

5. Ogden J, Tóth-Kischkat A, Schultheiss R. Principles of shock wave therapy. Clin Orthop Relat Res. 2001;387:8-17. https://doi.org/10.1097/00003086200106000-00003.

6. Vahdatpour B, Kiyani A, Dehghan FJABR. Effect of extracorporeal shock wave therapy on the treatment of patients with carpal tunnel syndrome. Adv Biomed Res. 2016;5(120). https://doi.org/10.4103/2277-9175.186983.

7. Paoloni M, Tavernese E, Cacchio A, et al. Extracorporeal shock wave therapy and ultrasound therapy improve pain and function in patients with carpal tunnel syndrome. A randomized controlled trial. Eur J Phys Rehabil Med. 2015:51(5):521-28.

8. Raissi G, Ghazaei F, Forogh B, Madani S, Daghaghzadeh A, Ahadi T. The effectiveness of radial extracorporeal shock waves for treatment of carpal tunnel syndrome: a randomized clinical trial. Ultrasound Med Biol. 2016; 43(2):453-60. https://doi.org/10.1016/j.ultrasmedbio.2016.08.022.

9. Kim JC, Jung SH, Lee S-U, Medicine SYLJ. Effect of extracorporeal shockwave therapy on carpal tunnel syndrome: a systematic review and meta-analysis of randomized controlled trials. Medicine (Baltimore). 2019;98(33):e16870. https://doi.org/10.1097/MD.0000000000016870.

10. Wu YT, Ke MJ, Chou YC, et al. Effect of radial shock wave therapy for carpal tunnel syndrome: a prospective randomized, double-blind, placebocontrolled trial. J Orthop Res. 2016;34(6):977-84. https://doi.org/10.1002/jor. 23113.

11. Xu D, Ma W, Jiang W, et al. A randomized controlled trial: comparing extracorporeal shock wave therapy versus local corticosteroid injection for the treatment of carpal tunnel syndrome. Int Orthop. 2020:44(1):141-6. https://doi.org/10.1007/s00264-019-04432-9. 
12. Atthakomol P, Manosroi W, Phanphaisarn A, Phrompaet S, lammatavee S, Tongprasert SJBMD. Comparison of single-dose radial extracorporeal shock wave and local corticosteroid injection for treatment of carpal tunnel syndrome including mid-term efficacy: a prospective randomized controlled trial. BMC Musculoskelet Disord. 2018;19(1):32. https://doi.org/10.1186/ s12891-018-1948-3.

13. Sweilam G, Elshahaly M, Hefny M. Extracorporeal shock wave therapy (ESWT) versus local steroids injection in the management of carpal tunnel syndrome. Biomed J Sci Tech Res. 2019;12. https://doi.org/10.26717/BJSTR. 2019.12.002281.

14. Seok H, Kim S. The effectiveness of extracorporeal shock wave therapy vs. local steroid injection for management of carpal tunnel syndrome a randomized controlled trial. Am J Phys Med Rehabil. 2013;92(4):327-34. https://doi.org/10.1097/PHM.0b013e31826edc7b.

15. Tao R, Wang J, Xia LJ, et al. Extracorporeal shock wave combined with median nerve injection in the treatment of early carpal tunnel syndrome. Chinese J Physical Med Rehabil. 2018;40(1):60-3. https://doi.org/10.3760/ cma.j.issn.0254-1424.2018.01.016.

16. Moher D, Liberati A, Tetzlaff J, et al. Methods of systematic reviews and meta-analysis preferred reporting items for systematic reviews and metaanalyses: the PRISMA statement. PLoS Medicine. 2009;8(7):336-41.https://doi. org/10.1371/joumal.pmedl000097.

17. Higgins JPT, Thomas J, Chandler J, Cumpston M, Li T, Page MJ, Welch VA (editors). Cochrane Handbook for Systematic Reviews of Interventions version 6.1. Cochrane. 2020. https://training.cochrane.org/ handbook.

18. Higgins JP, Altman DG, Gotzsche PC, et al. The Cochrane collaboration's tool for assessing risk of bias in randomised trials. BMJ. 2011;18(343):d5928 https://doi.org/10.1136/bmj.d5928.

19. Wang L. Guiding treatment for carpal tunnel syndrome. Phys Med Rehabil Clin N Am. 2018;29(4):751-60. https://doi.org/10.1016/j.pmr.2018.06.009.

20. Atroshi I. Steroid injection or wrist splint for first-time carpal tunnel syndrome? Lancet. 2018;392(10156):1383-4. https://doi.org/10.1016/S01406736(18)31929-9.

21. Ke M-J, Chen L-C, Chou Y-C, et al. The dose-dependent efficiency of radial shock wave therapy for patients with carpal tunnel syndrome: a prospective, randomized, single-blind, placebo-controlled trial. Sci Rep. 2016; 6:38344. https://doi.org/10.1038/srep38344.

22. Padua L, Padua R, Aprile I, Pasqualetti P, Tonali P. Carpal tunnel syndrome. Multiperspective follow-up of untreated carpal tunnel syndrome: a multicenter study. Neurology. 2001;56(11):1459-66. https://doi.org/10.1212/ WNL.56.11.1459

23. Wong S, Hui A. Corticosteroid injection for the treatment of carpal tunnel syndrome. Ann Rheum Dis. 2001;60(9):897.

24. Huisstede B, Manon S, Brink J, Franke T, Koes B, Hoogvliet P. The effectiveness of oral pain medication and corticosteroid injections for carpal tunnel syndrome-a systematic review. Arch Phys Med Rehabil. 2018;99(8): 1609-22. https://doi.org/10.1016/j.apmr.2018.03.003.

25. Mariotto S, de Prati AC, Cavalieri E, Amelio E, Marlinghaus E, Suzuki H. Extracorporeal shock wave therapy in inflammatory diseases: molecular mechanism that triggers anti-inflammatory action. Curr Med Chem. 2009;16: 2366-72. https://doi.org/10.2174/092986709788682119.

26. Ciampa A, de Prati AC, Amelio E, et al. Nitric oxide mediates antiinflammatory action of extracorporeal shock waves. FEBS letters. 2005; 579(30):6839-45. https://doi.org/10.1016/j.febslet.2005.11.023.

27. Mariotto S, Cavalieri E, Amelio E, et al. Extracorporeal shock waves: from lithotripsy to anti-inflammatory action by NO production. Nitric Oxide. 2005: 12(2):89-96. https://doi.org/10.1016/j.niox.2004.12.005.

28. Takahashi N, Wada Y, Ohtori S, Saisu T, Moriya H. Application of shock wave to rat skin decreases calcitonin gene-related peptide immunoreactivity in dorsal root ganglion neurons. Auton Neurosci. 2003;107(2):81-4. https://doi. org/10.1016/S1566-0702(03)00134-6

29. Mense $S$, Hoheisel U. Shock wave treatment improves nerve regeneration in the rat. Muscle Nerve. 2013;47(5):702-10. https://doi.org/10.1002/mus.23631.

30. Behr-Roussel D. Low-energy shock wave therapy ameliorates erectile dysfunction in a pelvic neurovascular injuries rat model. Transl Androl Urol. 2016;5(6):977-9. https://doi.org/10.21037/tau.2016.11.07.

31. Hausner $T$, Pajer $K$, Halát $G$, et al. Improved rate of peripheral nerve regeneration induced by extracorporeal shock wave treatment in the rat. Exp Neurol. 2012;236:363-70. https://doi.org/10.1016/j.expneurol.2012.04.019.
32. Ortiz-Corredor F, Calambas N, Mendoza-Pulido C, Galeano J, Diaz-Ruiz J, Delgado O. Factor analysis of Carpal Tunnel Syndrome Questionnaire in relation to nerve conduction studies. Cli Neurophysiol. 2011;122:2067-70. https://doi.org/10.1016/j.clinph.2011.02.030.

33. Gürsoy A, Kolukısa M, Yıldız G, Kocaman G, Celebi A, Koçer A. Relationship between electrodiagnostic severity and neuropathic pain assessed by the LANSS pain scale in carpal tunnel syndrome. Neuropsychiatr Dis Treat. 2013; 9:65-71. https://doi.org/10.2147/NDT.S38513.

34. Chan L, Turner J, Comstock B, et al. The relationship between electrodiagnostic findings and patient symptoms and function in carpal tunnel syndrome. Arch Phys Med Rehabil. 2007;88:19-24. https://doi.org/10. 1016/j.apmr.2006.10.013.

35. Kim P-T, Lee H-J, Kim T-G, Jeon I-H. Current approaches for carpal tunnel syndrome. Clin Orthop Surg. 2014;6:253-7.

36. Wu YT, Ke MJ, Ho TY, Li TY, Shen YP, Chen LC. Randomized double-blinded clinical trial of $5 \%$ dextrose versus triamcinolone injection for carpal tunnel syndrome patients. Ann Neurol. 2018;84(4):601-10.

37. Neustadt D. Complications of local corticosteroid injections. JAMA. 1981;246: 835-6.

38. Wild C, Khene M, Wanke S. Extracorporeal shock wave therapy in orthopedics. Assessment of an emerging health technology. Int J Technol Assess Health Care. 2000;16:199-209. https://doi.org/10.1017/ S0266462300161173.

39. Girlanda P, Dattola R, Venuto C, Mangiapane R, Nicolosi C, Messina C. Local steroid treatment in idiopathic carpal tunnel syndrome: short- and longterm efficacy. J Neurol. 1993;240:187-90. https://doi.org/10.1007/ BF00857526.

\section{Publisher's Note}

Springer Nature remains neutral with regard to jurisdictional claims in published maps and institutional affiliations.
Ready to submit your research? Choose BMC and benefit from:

- fast, convenient online submission

- thorough peer review by experienced researchers in your field

- rapid publication on acceptance

- support for research data, including large and complex data types

- gold Open Access which fosters wider collaboration and increased citations

- maximum visibility for your research: over $100 \mathrm{M}$ website views per year

At BMC, research is always in progress.

Learn more biomedcentral.com/submissions 